\title{
Introduction of advanced optics research to laboratory-experimental course for undergraduate teaching
}

Shiquan Tao, Zhuqing Jiang, Dayong Wang, Yuhong Wan

Shiquan Tao, Zhuqing Jiang, Dayong Wang, Yuhong Wan, "Introduction of advanced optics research to laboratory-experimental course for undergraduate teaching," Proc. SPIE 9664, Ninth International Topical Meeting on Education and Training in Optics and Photonics, 96640D (24 October 2005); doi: 10.1117/12.2207553

Event: Ninth International Topical Meeting on Education and Training in Optics and Photonics, 2005, Marseille, France 
Ref ETOP010

\title{
Introduction of advanced optics research to laboratory-experimental course for undergraduate teaching
}

Shiquan Tao, Zhuqing Jiang, Dayong Wang , and Yuhong Wan

College of Applied Sciences, Beijing University of Technology, Beijing 100022, China

Tel \& Fax: (+8610) 6739 1734, E-mail: shqtao@bjpu.edu.cn

\begin{abstract}
"Specialized Physics Experiments" is a significant laboratory-experimental course for Bachelor Degree in the Applied Physics discipline. In this paper, the introduction of advanced research in volume holographic storage to an integrated experiment of the course is presented. The results show that by introducing sophisticated experiment, which contains contemporary optics, mechanics, electronics, and computing, to the course experiments, the knowledge and technical abilities of undergraduate students can be effectively extended. This presents a model of combining scientific research projects with undergraduate teaching.
\end{abstract}

\section{Keywords}

laboratory-experimental course, specialized physical experiments, advanced research, volume holographic storage, undergraduate teaching

\section{Summary}

For the past several decades optics and photonics have been playing more and more important role in all sectors of modern society. Therefore, education and training in optics and photonics must meet the growing demands of widely-diverse fields of science, high technology, and modern industry, bring up students to the next-generation technicians, engineers and scientists. This is the main task of the Applied Physics discipline at Beijing University of Technology (formerly known as Beijing Polytechnic University). In order to realize this task, practice teaching, including laboratory experiments of various levels, are necessary in addition to lectures. "Specialized Physics Experiments" is a significant laboratory-experimental course for Bachelor Degree in the Applied Physics discipline, which is designed to deepen the understanding of specialized knowledge and develop the practice abilities and scientific quality of the students.

As a laboratory at a local university in a developing country, our Specialized Physics Course Lab was poorly equipped before the year of 2000. In the optics and photonics fields, it could only provide traditional experiments such as simple coherent optical processing (image subtraction, character recognition, etc.) with dated laboratory instruments. On the other hand, since 1996 when the University entered the 211 Project (a government - driven project to concentrate resources on some 100 key higheducation institutions and disciplines towards the 21 Century), it is considered to be among the top 3\% of the HEls in China. During the implementation of 211 Project, the University was more funded, and its scientific research and postgraduate teaching have been well developing. The research group of Optical Information Processing and Holography engaged in many research projects in the field of holographic storage and 
achieved good results. In this situation we tried to introduce advanced research to laboratory-experimental course for undergraduate teaching. The first attempt is to upgrade the Specialized Physics Experiments by adding an item, Angle Multiplexed Holographic Storage, into the course.

In this experiment the object beams carrying image information interfer with different angled reference beams, so volume holograms are recorded inside an iron doped $\mathrm{LiNbO} 3$ crystal. After recording the stored information is retrieved from the crystal. The recording and reply of the holograms are all controlled through computer interfaces. The purpose of this experiment is to enable students to get knowledge of the mechanism of holographic information storage with photorefractive crystals, to master the computerbased experimental method in holography, and to get experience with state-of-the-art optoelectronic devices. Figure 1 shows the experimental setup.

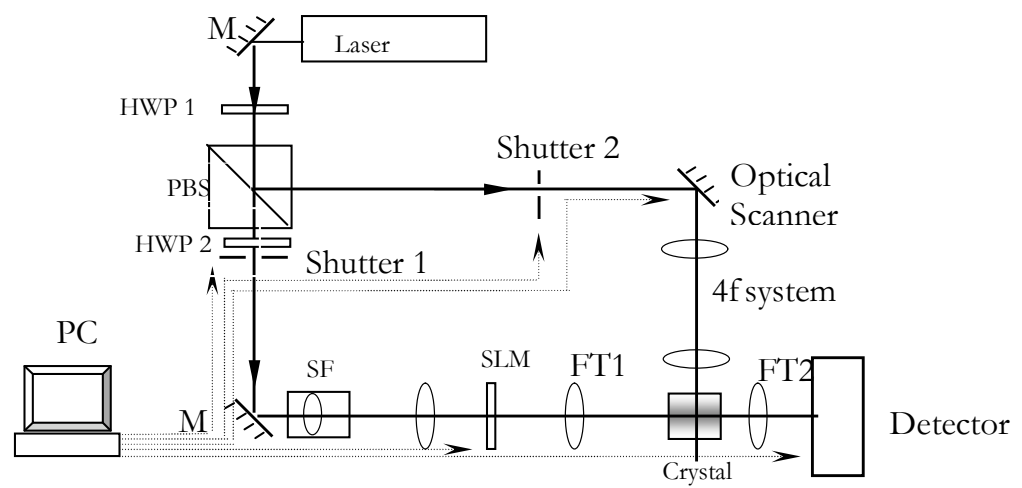

Fig.1 A schematic diagram of the experimental setup. M: mirror, HWP: half-wave plate, PBS: polarization beam splitter, SF: spatial filter, SLM: spatial light modulator, FT: Fourier transform lens, Detector: image detector array (CCD) or power meter, PC: computer. Solid lines indicate light beams, and dashed lines indicate remote control through computer interfaces.

According to the experiment guide, the students are required to do the following:

1. Record a volume holographic grating in a Fe:LiNbO3 crystal by the two-wave mixing configuration, and acquire the kinetics of grating formation by means of computer-aided sampling of the diffracted power;

2. Process the data from the acquired writing curve, and calculate the holographic parameters of the crystal such as maximum diffraction efficiency, writing time constant, saturated index modulation, and sensitivity;

3. Read out the recorded grating by scanning the reference beam, and acquire the diffracted power as a function of the readout angle;

4. Process the data from the scanned curve and obtain the angular selectivity of the volume hologram in the crystal, compare the result with the theoretical expectation, and evaluate the storage ability of the crystal for angle multiplexing of holograms; 
5. Record holograms of images displayed on the electric-SLM, and sample the reconstructed images through a CCD detector, observe .the fidelity of the retrieved images.

It is obvious that this is an integrated experiment consisting of training in information optics, optoelectronics, electronics, mechanics and computing. Through the experiment the students get experience with opto-electric conversion, A/D and D/A conversion, data and image acquisition, and relevant state-of-the-art devices such as the high-resolution liquid crystal SLM, and the high-resolution, low-noise CCD. Thus the knowledge and technical abilities of undergraduate students can be effectively extended. Furthermore, course experiments like this evoked the interests of students in optics and photonics, encouraged some of them to pursue further study in these fields. So, the introduction of this experiment is beneficial to both the undergraduate and the postgraduate teaching. Also, this presents a modal for the incorporation of scientific research into teaching, and the sharing of the educational resources of postgraduate teaching with undergraduate teaching. 